\title{
Nature of the Isotope Effect on Transport in Tokamaks
}

\author{
M. Z. Tokar, D. Kalupin, and B. Unterberg \\ Institut für Plasmaphysik, Forschungszentrum Jülich GmbH, Association FZJ-Euratom, 52425, Jülich, Germany
}

(Received 5 November 2003; published 28 May 2004)

\begin{abstract}
The reduction of energy and particle losses with the increasing mass of the hydrogen isotope is more pronounced under conditions of improved confinement when the dominant ion temperature gradient instability is suppressed and other channels of anomalous transport are of importance. In this Letter, we reconsider the dissipative trapped electron (DTE) instability by taking into account finite Larmor radius effects in the analysis of the ion response to perturbations. By applying the improved mixing length approximation in order to estimate the transport coefficients, it is demonstrated that DTE contribution is intrinsically dependent on the isotope mass and provides a plausible explanation for the isotope effect. Contrary to the common belief, it is shown that the DTE turbulence may be of importance for reactor plasmas of low collisionality.
\end{abstract}

DOI: 10.1103/PhysRevLett.92.215001

PACS numbers: 52.35.Py, 52.55.Fa

As observed in practically all tokamaks, the energy and particle confinement improves with increasing atomic weight of the isotope used, from hydrogen to deuterium to tritium (see, e.g., Refs. [1-3]). This fact is of crucial importance for the performance of future thermonuclear reactors, which will operate with a mixture of deuterium and tritium rather than present devices which normally use either hydrogen or deuterium. However, in spite of its fundamental significance, the isotope effect is still one of the least understood phenomena in the physics of tokamak transport.

Analysis of the experimental database shows that the isotope effect on confinement depends essentially on the mode of tokamak operation. Thus, the most recent scalings for the energy confinement time $\tau_{E}$ [4] show in low $(L)$ mode a rather moderate, as $A_{i}^{0.2}$, increase with the atomic weight $A_{i}$. Plasma states, where improved confinement is accompanied by peaked density profiles, reveal a significantly stronger mass dependence. For example, in supershots in Tokamak Fusion Test Reactor (TFTR) $\tau_{E}$ scales as $A_{i}^{0.85}$ [2], in pellet fueled plasmas in ASDEX [1], in the radiation improved (RI) mode in TEXTOR [5] and in discharges with improved Ohmic confinement [1], $\tau_{E} \sim A_{i}^{0.5}$. The latter dependence is also characteristic of the edge transport barrier in the $H$ mode [3]. Conversely, in the core of $H$-mode plasmas the confinement drops as $A_{i}^{-0.2}$ [3] and the overall $H$-mode isotope effect is similar to that in the $L$ mode [4].

A pattern in this puzzling picture can be found by noting that the strongest improvement with $A_{i}$ takes place if the ion temperature gradient (ITG) instability, which is commonly considered as the main source of anomalous transport [6], is suppressed. This is the case both for the edge barrier in the $H$ mode and for other regimes of improved confinement where ITG is subdued by the density gradient in a large part of the plasma volume (see, e.g., analysis of the $L-\mathrm{RI}$ transition in Refs. [7,8]). On the contrary, under conditions where the transport is dominated by ITG modes the confinement deteriorates with increasing $A_{i}$. For the case of the plasma core in the $H$ mode, this fact was explained in Ref. [9] by applying the mixing length approximation (MLA) $[6,10,11]$, in which $\gamma_{\max } / k_{\max }^{2}$ is used as an estimate for transport coefficients. Here $\gamma_{\max }$ is the maximum value of the instability growth rate $\gamma$ considered as a function of the perpendicular wave number $k$ and $k_{\max }$ is the $k$-value where $\gamma_{\max }$ is reached. For the ITG instability both $\gamma_{\max }$ and $k_{\max }$ vary as $\sim A_{i}^{-0.5}[6,12]$ and the characteristic heat diffusivity $\chi \sim A_{i}^{0.5}$. Since $\tau_{E} \sim 1 / \chi$, the scaling above is in qualitative agreement with the decrease of the core confinement in the $H$ mode with increasing isotope atomic weight. The same result follows from the so-called improved mixing length approximation (IMLA) [6,13], which takes into account the phase shift between perturbations of the particle density and radial velocity.

On this basis one can suggest that the positive isotope effect on confinement is rooted in the influence of the ion mass on the transport channels that dominate when ITG turbulence is reduced or suppressed. In Refs. [14,15], instabilities driven by impurities were analyzed, and it was demonstrated that their growth rates are reduced with increasing $A_{i}$. However, the importance of these instabilities for tokamak confinement has been cast in doubt by the discovery of plasma states where anomalous transport is reduced due to the deliberate seeding of impurities [16]. A positive isotope effect was found also in numerical modeling of collisional drift turbulence [17]. However, this kind of turbulence is localized at the very plasma edge and cannot explain the modification of profiles in the central region with varying isotope composition.

In the plasma core, dissipative trapped electron (DTE) modes [11] are often considered as the most important under conditions where ITG activity is absent. The DTE instability is caused by the specific velocity dependence 
of the collision frequency of trapped particles [11,18-20]. DTE induced transport has been identified as the main channel of particle and energy losses in the linear Ohmic [21], improved Ohmic [22], and RI [8] modes of confinement. Its enhancement is believed to be the main cause of confinement deterioration in the RI mode when hydrogen puffing is applied instead of deuterium puffing [23]. In Ref. [23], this was assumed to be caused by the reduction of the ion effective charge.

In this Letter it is shown that in addition to the latter possibility the DTE transport exhibits an explicit ion mass dependence. This arises if finite Larmor radius corrections are taken into account in the description of the ion component and one makes use of the improved mixing length approximation in estimating the transport coefficients. The IMLA has been firmly validated in Ref. [11] by demonstrating that its predictions are similar to those from strong turbulence models. The results obtained below provide an explanation for the isotope effect on transport both in collision dominated plasmas, e.g., in RI mode, and under reactor conditions of low collisionality.

Consider a hydrogen isotope plasma, characterized by stationary radial profiles of density and temperature (assumed to be equal for electrons and ions), $n(r)$ and $T(r)$, respectively. The toroidal magnetic field decays with the distance $R$ from the tokamak axis, $B=B_{0} \frac{R_{0}}{R}$, where $B_{0}$ is the magnetic field at the plasma axis situated at radius $R_{0}$. A perturbation of the electric potential, $\tilde{\varphi} \sim$ $\exp (i k y-i \omega t)$, where $\omega$ is the complex frequency and $k$ the wave vector in the direction perpendicular both to the magnetic field and to the radial direction, induces drift motion of plasma ions. As a result the ion density is also perturbed [6]:

$$
\frac{\tilde{n}_{i}}{n}=\frac{\omega_{*}-\omega_{D}-\xi\left(\omega+\omega_{*}+\omega_{T}\right)}{\omega+\omega_{D}} \frac{e \tilde{\varphi}}{T},
$$

where $\omega_{*, T}=\zeta c_{s} / L_{n, T}$ and $\omega_{D}=2 \zeta c_{s} / R_{0}$ are characteristic drift frequencies with $\zeta=k \rho_{p}$ being the dimensionless wave number, $\rho_{p}=c \sqrt{m_{p} T} /(e B)$ and $c_{s}=\sqrt{T / m_{p}}$ are the proton Larmor radius and sound velocity, $L_{n}=$ $-\frac{d r}{d \ln n}, L_{T}=-\frac{d r}{d \ln T}$, and $\xi=A_{i} \zeta^{2}$. A similar relation supplemented by the effect of temperature perturbations, is commonly used in ITG models [6]. Conversely, the relation $\frac{\tilde{n}_{i}}{n}=\frac{\omega_{*}}{\omega} \frac{e \tilde{\varphi}}{T}$, which follows from Eq. (1) with the assumptions $\omega_{D} \ll \omega_{*}$ and $\xi=0$, is normally adopted in the DTE instability analysis [11,18-20]. Through the factor $\xi$, Eq. (1) includes the ion mass effect, which stems from damping of drift motion by gyroviscosity [24].

Kinetic analysis is the best approach to describe trapped electrons. However, a detailed fluid description gives similar results [19]. By taking into account both trapped electrons and particles freely circulating on magnetic surfaces, the following relation can be obtained [19]:

$$
\frac{\tilde{n}_{e}}{n}=\left(1+f_{\mathrm{tr}} \frac{\omega_{*}-\omega+i \nu_{\mathrm{tr}} \frac{\omega_{T}}{\omega-\omega_{D}+i \nu_{\mathrm{tr}}}}{\omega+i \nu_{\mathrm{tr}}}\right) \frac{e \tilde{\varphi}}{T},
$$

where $f_{\mathrm{tr}}$ and $\nu_{\mathrm{tr}}=\nu_{e} \frac{R_{0}}{r}$ are the fraction and effective collision frequency of trapped electrons and $\nu_{e}$ is the collision frequency of thermal electrons.

The dispersion relation between $\omega$ and $\zeta$ follows from the quasineutrality condition $\tilde{n}_{i}=\tilde{n}_{e}$. This results in the following set of algebraic equations for the real frequency $\omega_{r} \equiv \operatorname{Re}(\omega)$ and the growth rate $\gamma \equiv \operatorname{Im}(\omega)$ of the perturbations:

$$
\omega_{r}=\omega_{0}-\frac{f_{\text {tr }}}{1+\xi} \operatorname{Im} \Omega, \quad \gamma=\frac{f_{\text {tr }}}{1+\xi} \operatorname{Re} \Omega,
$$

where

$$
\begin{gathered}
\omega_{0}=\frac{\omega_{*}-2 \omega_{D}-\xi\left(\omega_{*}+\omega_{T}\right)}{1+\xi} \\
\Omega=\frac{\omega+\omega_{D}}{\nu_{\mathrm{tr}}-i \omega}\left(\omega_{*}-\omega+\frac{i \nu_{\mathrm{tr}} \omega_{T}}{\omega-\omega_{D}+i \nu_{\mathrm{tr}}}\right) .
\end{gathered}
$$

Figure 1 shows the $\zeta$ dependences of the growth rate and real frequency of the DTE instability for $\mathrm{H}^{+}, \mathrm{D}^{+}$, and $T^{+}$plasmas computed from the numerical solution of Eqs. (3) for TEXTOR RI-mode conditions [8]: $R_{0}=$ $1.75 \mathrm{~m}, r=2 / 3 a \approx 0.3 \mathrm{~m}, B_{T}=2.25 \mathrm{~T}, T=0.5 \mathrm{keV}$, $n=5 \times 10^{19} \mathrm{~m}^{-3}, L_{n, T}=0.2 \mathrm{~m}$.

It can be seen that the maximum growth rate $\gamma_{\max }$, the corresponding wave number $k_{\max }=\zeta_{\max } / \rho_{p}$, and the real frequency $\omega_{r \max }$ at $k_{\max }$ decrease with increasing $A_{i}$, but to a different degree. This leads to a significant variation

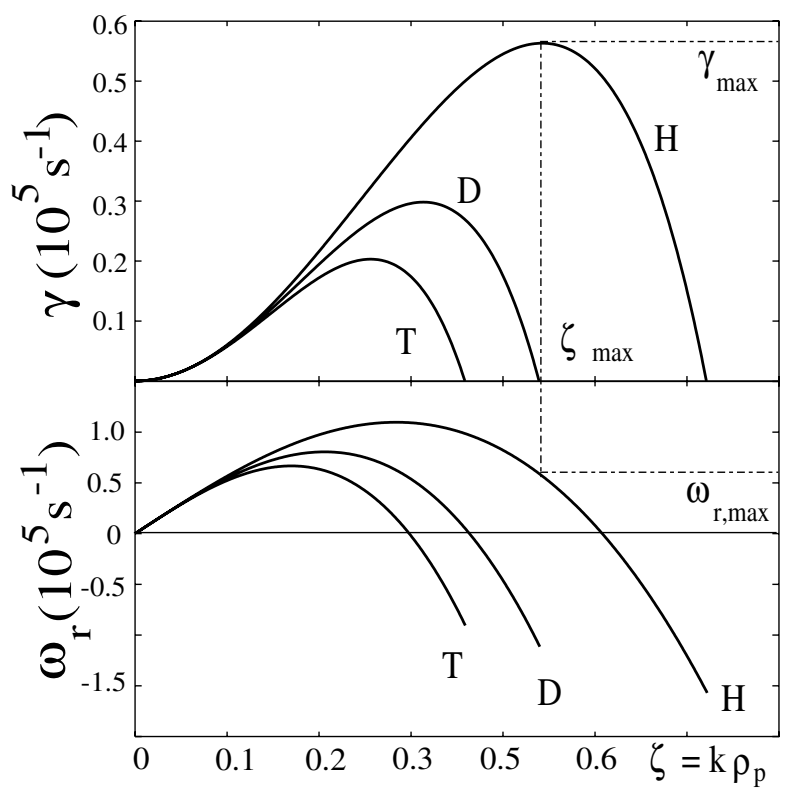

FIG. 1. The $\zeta(k)$ dependences of the growth rate $\gamma$ and the real frequency $\omega_{r}$ for unstable DTE modes in plasmas of different hydrogen isotopes computed for RI-mode conditions in TEXTOR. 
of the heat diffusivity computed in the improved mixing length approximation $[6,13]$ :

$$
\chi \approx C \frac{\gamma_{\max }}{k_{\max }^{2}} \frac{\gamma_{\max }^{2}}{\omega_{r \max }^{2}+\gamma_{\max }^{2}} .
$$

Here the numerical factor $C=2.88 \sqrt{1+2 q^{2}}$, with $q$ being the safety factor, was introduced according to Refs. [13,18]. Figure 2 shows the $A_{i}$ dependence of the heat diffusivity computed for $q=2$ and normalized to the gyro-Bohm coefficient $\chi_{g B}=c_{s} \rho_{p}^{2} / L_{n}$.

In order to demonstrate that the IMLA factor $\gamma_{\max }^{2} /\left(\omega_{r \max }^{2}+\gamma_{\max }^{2}\right)$ is actually responsible for the $A_{i}$ dependence of $\chi$, we estimate $k_{\max }, \gamma_{\max }, \omega_{r \max }$ from an approximate analytical solution of Eqs. (3). It can be obtained if one takes into account that relatively cold plasmas in TEXTOR are dominated by collisions, i.e., $\omega_{D}, \omega_{*}, \omega_{T} \ll \nu_{\text {tr }} \approx 10^{6} \mathrm{~s}^{-1}$ in the whole $\zeta$ range of positive $\gamma$. In this case we get

$$
\omega_{r} \approx \omega_{0}, \quad \gamma \approx \gamma_{0} / A_{i} \times g
$$

with $\gamma_{0}=f_{\text {tr }} c_{s}^{2} /\left(\nu_{\text {tr }} L_{n}^{2}\right)$ and

$$
g(\xi, \eta, \varepsilon)=\xi \frac{[\eta+2 \varepsilon+2 \xi(1+\eta)][1-\varepsilon-\xi(1+\eta-\varepsilon)]}{(1+\xi)^{2}},
$$

where $\varepsilon=2 L_{n} / R$ and $\eta=L_{n} / L_{T}$. The function $g$ reduces to zero at $\xi=0$ and $\xi=(1-\varepsilon) /(1+\eta-\varepsilon)$ and is positive between these values. For the parameters adopted above, one has $\varepsilon=0.23$ and $\eta=1$, and $g$ approaches the maximum $g_{\max } \approx 0.132$ at $\xi=\xi_{\max } \approx 0.22$. Finally,

$$
\begin{gathered}
\zeta_{\max } \approx \sqrt{\frac{\xi_{\max }}{A_{i}}}, \quad \gamma_{\max }=\frac{c_{s}^{2} f_{\mathrm{tr}}}{L_{n}^{2} \nu_{\mathrm{tr}}} \frac{g_{\max }}{A_{i}}, \\
\omega_{r \max } \approx \frac{c_{s}}{L_{n}} \frac{f_{\max }}{\sqrt{A_{i}}}
\end{gathered}
$$

with $f_{\max }=\sqrt{\xi_{\max }}\left[1-2 \varepsilon-\xi_{\max }(1+\eta)\right] /\left(1+\xi_{\max }\right)$.

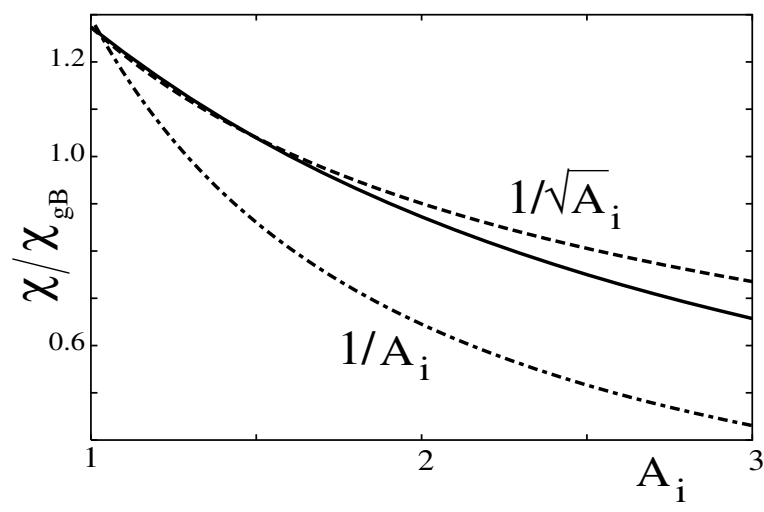

FIG. 2. The isotope mass dependence of DTE heat diffusivity (solid curve) computed according to Eq. (4) for TEXTOR conditions.
As one can see, $\gamma_{\max } / k_{\max }^{2}$ is mass independent and the $A_{i}$ dependence of $\chi$ is, indeed, governed only by the IMLA factor, i.e., by the relation between $\gamma_{\max }$ and $\omega_{r \max }$. If $\omega_{r \max } \ll \gamma_{\max }$ and the MLA is relevant, the transport coefficient does not reveal any isotope effect. In the opposite limit, $\omega_{r \max } \gg \gamma_{\max }$, we get $\chi \sim 1 / A_{i}$. The numerically found dependence in Fig. 2 lies between these two limiting cases.

Coulomb collisions of trapped particles play a key role for the excitation of DTE instability. In a future fusion reactor, e.g., ITER [4], the collision frequency will be much smaller than in the case of the TEXTOR RI mode discussed above. For ITER-FEAT parameters in the inductive operation stage [25], $R_{0}=6.2 \mathrm{~m}, a=2 \mathrm{~m}$, volume averaged $T=9 \mathrm{keV}$, and $n=10^{20} \mathrm{~m}^{-3}$, we get $\nu_{\mathrm{tr}} \approx 2.6 \times 10^{4} \mathrm{~s}^{-1}$, i.e., a value by a factor of 50 smaller than in the RI mode. Therefore, one might argue that DTE turbulence will be unimportant under reactor conditions. However, what actually matters is the comparison of $\nu_{\text {tr }}$ with characteristic drift frequencies $\omega_{*, T}$ and $\omega_{D}$, which are proportional to $\zeta$. Thus, there is always a range of relatively small wave numbers, for which even such a low collisionality can lead to DTE instability. Figure 3 shows the results of calculations done by using Eqs. (3) for ITER with $B_{T}=5.3 \mathrm{~T}, L_{T}=1.3 \mathrm{~m}$, and $L_{n}=2 L_{T}$. First, they demonstrate that under reactor conditions DTE turbulence leads to a transport contribution of the order of $0.5 \chi_{g B} \approx 0.5 \mathrm{~m}^{2} / \mathrm{s}$; i.e., it can be one of the most important channels for anomalous energy losses. Second, this contribution exhibits a very pronounced isotope effect leading to a reduction of $\chi$ by a factor of 2 when going from hydrogen to a deuterium-tritium mixture. Third, the maximum growth rate and corresponding real frequency are comparable to $\nu_{\text {tr }}$ and, therefore, the qualitative analysis above leading to estimates (6) and (7) is not applicable. This is clearly seen in Fig. 4 comparing the growth rate found for a $D-T$ mixture with $A_{i}=2.5$

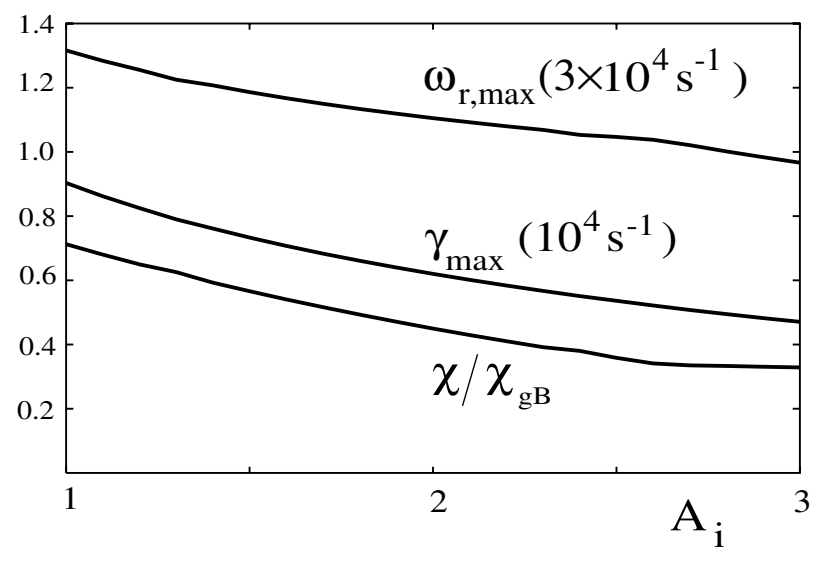

FIG. 3. The isotope mass dependence of the maximum growth rate, the corresponding real frequency and DTE heat diffusivity computed for plasma conditions in ITER-FEAT. The corresponding DTE perturbations have $\zeta=k \rho_{p}$ of 0.1 . 


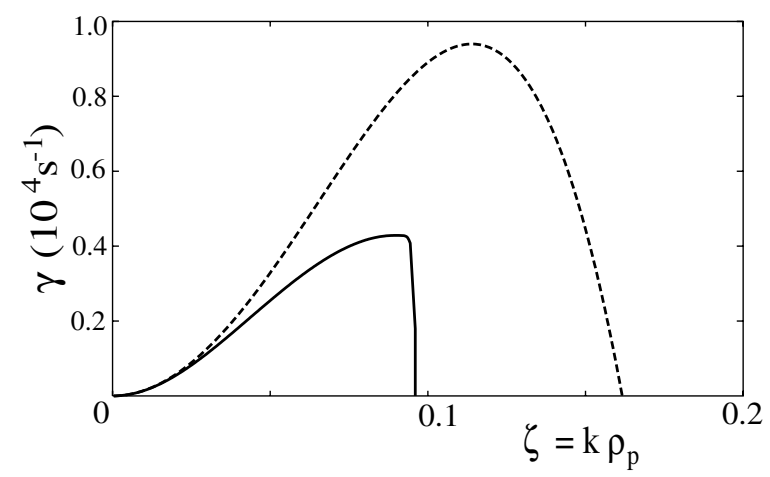

FIG. 4. The $\zeta(k)$ dependences of the DTE growth rate computed for ITER-FEAT plasmas with effective $A_{i}=2.5$ from a numerical solution of Eqs. (3) (solid curve) and calculated according to Eqs. (6) and (7) obtained in the limit of large collision frequency (broken curve).

from a numerical solution of Eqs. (3) and that resulting from Eqs. (5). The growth rate of DTE perturbations is, indeed, significantly, by a factor of 5, smaller than for the TEXTOR conditions. However, $\zeta_{\max }$ also noticeably decreases, and the contribution of DTE modes to the transport in the reactor case is comparable to that in TEXTOR.

Dissipative trapped electron modes make a significant contribution to energy losses in the TEXTOR RI mode where electron collisionality is very high. Although with decreasing collisionality the growth rate of the most unstable DTE perturbations becomes smaller, their wave length increases. Therefore, DTE turbulence can be of importance also under reactor conditions where the collisionality is low. In both limiting cases this contribution to anomalous transport becomes smaller with increasing isotope mass $A_{i}$. This dependence arises if in order to estimate $\chi$ the improved mixing length approximation, taking into account the phase shift between perturbations of density and radial particle velocity, is applied. This shift is determined both by the real frequency and by the growth rate of unstable perturbations. Since for DTE modes $\omega_{r}$ is governed by the ion dynamics and the response of electrons is of importance for $\gamma$, they vary in a different way with $A_{i}$. This explains the mass dependence of $\chi$. The latter is qualitatively different from that in the ITG-instability case where both the growth rate and real frequency are determined by the ion behavior and $\gamma_{\max } \sim \omega_{r \max } \sim 1 / \sqrt{A_{i}}$. Therefore, the phase shift factor is independent of $A_{i}$ and both MLA and IMLA give the same scaling for transport coefficients, increasing with the isotope mass [9].

[1] M. Bessendodt-Weberpals et al., Nucl. Fusion 33, 1205 (1993).

[2] R. J. Hawryluk, Rev. Mod. Phys. 70, 537 (1998).

[3] J. Jacquinot and the JET Team, Plasma Phys. Controlled Fusion 41, A13 (1999).

[4] ITER Physics Expert Groups on Confinement and Transport and Confinement Modelling and Databases, ITER Physics Basic Editors, Nucl. Fusion 39, 2175 (1999).

[5] G. Van Wassenhove et al., in Proceedings of the 29th EPS Conference on Plasma Physics and Controlled Fusion, Montreux, 2002 (Institute of Physics, Bristol, U.K., 2002), Vol. 26B, p. 2.057.

[6] J. Weiland, Collective Modes in Inhomogeneous Plasma (Institute of Physics Publishing, Bristol, 2000).

[7] M. Z. Tokar et al., Plasma Phys. Controlled Fusion 41, L9 (1999).

[8] M. Z. Tokar et al., Phys. Rev. Lett. 84, 895 (2000).

[9] G. Bateman, Phys. Plasmas 6, 4607 (1999).

[10] W. Horton, Rev. Mod. Phys. 71, 735 (1999).

[11] B. B. Kadomtsev and O. P. Pogutse, Sov. Phys. Dokl. 14, 470 (1969).

[12] J. Q. Dong et al., Phys. Plasmas 1, 3635 (1994).

[13] J.W. Connor and O. P. Pogutse, Plasma Phys. Controlled Fusion 43, 155 (2001).

[14] B. Coppi, in Plasma Physics Controlled Nuclear Fusion Research, Proceedings of the 13th International Conference, Washington, DC, 1990 (IAEA, Vienna, 1991), Vol. 2, p. 413.

[15] R. R. Dominguez, Nucl. Fusion 31, 2063 (1991).

[16] J. Ongena et al., Phys. Plasmas 8, 2188 (2001).

[17] B. D. Scott, Phys. Fluids B 4, 2468 (1992).

[18] A. Rogister, Phys. Plasmas 2, 2729 (1995).

[19] J. Nilsson and J. Weiland, Nucl. Fusion 34, 803 (1994).

[20] J. Wesson, Tokamaks (Clarendon Press, Oxford, 1997), 2nd ed.

[21] R. R. Dominguez and R. E. Waltz, Nucl. Fusion 27, 65 (1987).

[22] F. X. Söldner et al., Phys. Rev. Lett. 61, 1105 (1988).

[23] D. Kalupin et al., Plasma Phys. Controlled Fusion 45, 1501 (2003).

[24] S. I. Braginski, in Reviews of Plasma Physics, edited by M. A. Leontovitch (Consultants Bureau, New York, 1965), Vol. 1, p. 205.

[25] Y. Shimomura et al., Nucl. Fusion 41, 309 (2001). 